\title{
Uniform Convergence Rates for Halfspace Depth
}

\author{
Michael A. Burr ${ }^{1}$, Robert Fabrizio \\ Department of Mathematical Sciences, Clemson University, Clemson, SC 29634
}

\begin{abstract}
Data depth functions are a generalization of one-dimensional order statistics and medians to real spaces of dimension greater than one; in particular, a data depth function quantifies the centrality of a point with respect to a data set or a probability distribution. One of the most commonly studied data depth functions is halfspace depth. Halfspace depth is of interest to computational geometers because it is highly geometric, and it is of interest to statisticians because it shares many desirable theoretical properties with the one-dimensional median. It is known that as the sample size increases, the halfspace depth for a sample converges to the halfspace depth for the underlying distribution, almost surely. In this paper, we use the geometry and structure of halfspace depth to reduce a high-dimensional problem into many one-dimensional problems. This bound requires only mild assumptions on the distribution, and it leads to an improved convergence rate when the underlying distribution decays exponentially, i.e., the probability that a sample point has magnitude at least $R$ is $O\left(\exp \left(-\lambda R^{2} / 2\right)\right)$. We also provide examples and show that our bounds are tight.
\end{abstract}

Keywords: Data depth, Halfspace depth, Convergence, Glivenko-Cantelli, Vapnik-Červonenkis

\section{Introduction}

Data depth functions generalize order statistics and the median in one dimension to higher dimensions; in particular, they provide quantitative measures of the centrality of a point relative to a data set or a probability distribution (see [16], [15], and [20] for surveys). For these functions, larger values indicate that a point is deeper or more central with respect to a data set or distribution. The point with largest depth is defined to be the median of a data set or distribution. For data sets, data depth functions are typically defined in terms of the geometry of the data, and they reflect the geometric combinatorics of the data set.

Halfspace depth is a data depth measure that was first introduced by Hodges [13] and Tukey [22]. Halfspace depth has attracted the interest of computational geometers because of its strong geometric properties (see [1], [3], and [11]), and it is of interest to statisticians because it shares many theoretical properties with the one-dimensional median [24]. We recall the definition of halfspace depth for distributions and data sets, briefly using the notation $\mathcal{H}(q)$ for the set of halfspaces in $\mathbb{R}^{d}$ containing $q \in \mathbb{R}^{d}$.

Email addresses: burr2@clemson.edu (Michael A. Burr), rfabriz@clemson.edu (Robert Fabrizio)

${ }^{1}$ This work was partially supported by grants from the Simons Foundation (\#282399 to Michael Burr) and the NSF (\#CCF-1527193) 
Definition 1.1. Let $X$ be an $\mathbb{R}^{d}$-valued random variable. For a point $q \in \mathbb{R}^{d}$, the halfspace depth of $q$ with respect to $X$ is the minimum probability that $X \in H$, where $H$ is taken over all halfspaces $H$ in $\mathbb{R}^{d}$ containing $q$ :

$$
\operatorname{HD}(q ; X)=\min _{H \in \mathcal{H}(q)} \operatorname{Pr}(X \in H) .
$$

Let $X^{(n)}=\left(X_{1}, \cdots, X_{n}\right)$ be a finite sample of $n$ points in $\mathbb{R}^{d}$. For a point $q \in \mathbb{R}^{d}$, the halfspace depth of $q$ with respect to $X^{(n)}$ is the minimum fraction of sample points in $H$, where $H$ is taken over all halfspaces $H$ in $\mathbb{R}^{d}$ containing $q$ :

$$
\operatorname{HD}\left(q ; X^{(n)}\right)=n^{-1} \min _{H \in \mathcal{H}(q)} \#\left\{X^{(n)} \cap H\right\} .
$$

Notation 1.2. Throughout the remainder of this paper, we denote a positive integer by $d$ and an $\mathbb{R}^{d}$-valued random variable by $X$. Moreover, we let $X_{1}, X_{2}, \ldots$ be a sequence of iid random variables equal in distribution to $X$. We denote a finite sample by $X^{(n)}=\left(X_{1}, \cdots, X_{n}\right)$, consisting of the first $n$ elements of this sequence.

It is shown in [8] that, as $n \rightarrow \infty$,

$$
\sup _{q \in \mathbb{R}^{d}}\left|\operatorname{HD}(q ; X)-\operatorname{HD}\left(q ; X^{(n)}\right)\right| \rightarrow 0 \quad \text { a.s. }
$$

In this paper, we use a previously overlooked approach using the geometry of halfspace depth to improve explicit bounds on the rate of convergence of this limit. In particular, we reduce a high dimensional problem to many one-dimensional problems. The main advance of this paper is that we show the following:

Theorem 1.3. Suppose that $\operatorname{Pr}(\|X\|>R)$ decays exponentially and $X$ satisfies mild continuity assumptions (see Section 4). Fix $\varepsilon>0$. Then there is a constant $C$ such that for $n$ sufficiently large,

$$
\operatorname{Pr}\left(\sup _{q \in \mathbb{R}^{d}}\left|\operatorname{HD}(q ; X)-\operatorname{HD}\left(q ; X^{(n)}\right)\right| \geq \varepsilon\right) \leq C n^{\frac{3}{2}(d-1)} e^{-2 n \varepsilon^{2}} .
$$

This theorem represents an improvement by a factor of $n^{\frac{1}{2} d+\frac{7}{2}}$ over the previous bound (when the mild conditions in the theorem are satisfied).

The remainder of this paper is organized as follows: In Section 2, we review the previous work on the convergence of halfspace depth. In Section 3, we define appropriate notation and cite results from both discrete geometry and probability theory. We prove the main convergence bounds in Section 4. In Section 5, we prove the main theorem and illustrate the convergence bounds with a few examples and special cases, and, finally, we conclude in Section 6 .

\section{Previous Convergence Results}

In [8], the authors prove that for all $q \in \mathbb{R}^{d}, \operatorname{HD}\left(q ; X^{(n)}\right) \rightarrow \operatorname{HD}(q ; X)$ almost surely as $n \rightarrow \infty$ (see also [10], [12], [25], [14], and [17] for additional, related convergence results). This 
result is proved by observing that the collection of all halfspaces in $\mathbb{R}^{d}$ satisfies the GlivenkoCantelli property (for additional details, see [21]), so that, uniformly for all halfspaces $H$, $n^{-1} \#\left\{X^{(n)} \cap H\right\} \rightarrow \operatorname{Pr}(X \in H)$ a.s. as $n \rightarrow \infty$.

The convergence given by the Glivenko-Cantelli property is strengthened by observing that the set of halfspaces are a Vapnik-Cervonenkis class. In particular, it is shown in [9] that halfspaces in $\mathbb{R}^{d}$ cannot shatter sets of size $d+2$ (sets of size $d+1$ consisting of the corners of a convex polygon can be shattered). We write $m(n)$ for the maximum number of subsets formed by intersecting finite sets of $n$ points with halfspaces in $\mathbb{R}^{d}$. Then, in [23] and [5], the following result is proved:

Proposition 2.1 (See [21, Chapter 26]). Let $\mathcal{H}$ be the set of all halfspaces in $\mathbb{R}^{d}$ and suppose that $\varepsilon>0$. Define $m$ to be the function as described above. Then, for $n$ sufficiently large,

(1) $\operatorname{Pr}\left(\sup _{H \in \mathcal{H}}\left|n^{-1} \#\left\{X^{(n)} \cap H\right\}-\operatorname{Pr}(X \in H)\right| \geq \varepsilon\right) \leq 4 m(2 n) e^{-n \varepsilon^{2} / 8}$.

(2) $\operatorname{Pr}\left(\sup _{H \in \mathcal{H}}\left|n^{-1} \#\left\{X^{(n)} \cap H\right\}-\operatorname{Pr}(X \in H)\right| \geq \varepsilon\right) \leq 4 m\left(n^{2}\right) e^{4 \varepsilon+4 \varepsilon^{2}} e^{-2 n \varepsilon^{2}}$.

Moreover, $m(r) \leq \frac{3 r^{d+1}}{2(d+1) !}$.

In this paper, we improve these bounds on the error by increasing the magnitude of the exponent in the exponential or decreasing the degree of the polynomial coefficient.

\section{Notation and Prior Results}

Since data depth combines discrete geometry with probability and statistics, our main result requires results from all of these fields. In this section, for convenience, we collect a few theorems and notations that are used in the remainder of the paper.

\subsection{Parametrizing Halfspaces}

We begin this section by observing that every halfspace can be parametrized by an outward pointing normal vector and a signed distance from the origin. In particular, we parametrize halfspaces by points in $\mathbb{S}^{d-1} \times \mathbb{R}$, where $\mathbb{S}^{d-1}$ is the $(d-1)$-dimensional sphere:

Notation 3.1. Let $\theta \in \mathbb{S}^{d-1}$; define $u_{\theta}$ to be the vector in $\mathbb{R}^{d}$ pointing in the direction of $\theta, \ell_{\theta}$ to be the line through the origin in the direction of $\theta$, and $\pi_{\theta}: \mathbb{R}^{d} \rightarrow \ell_{\theta}$ to be the orthogonal projection of $\mathbb{R}^{d}$ onto $\ell_{\theta}$. Moreover, for a point $p \in \mathbb{R}^{d}$, define $d_{\theta}(p)=\left\langle p, u_{\theta}\right\rangle$ (where $\langle\cdot, \cdot\rangle$ is the standard inner product in $\mathbb{R}^{d}$ ) to be the signed length of $p$ in the direction of $u_{\theta}$. In other words, $\pi_{\theta}(p)=d_{\theta}(p) u_{\theta}$.

Using this notation, we define probability distributions and finite samples on $\mathbb{R}$ corresponding to each direction in $\mathbb{S}^{d-1}$ :

Notation 3.2. For each $\theta \in \mathbb{S}^{n-1}, X_{\theta}=d_{\theta}(X)$ is an $\mathbb{R}$-valued random variable, and $F_{\theta}$ is the cdf for this variable, i.e., $F_{\theta}(t)=\operatorname{Pr}\left(X_{\theta} \leq t\right)$. Similarly, $F_{n, \theta}$ is the empirical cdf for the points of $X^{(n)}$ in the direction of $\theta$, i.e., $F_{n, \theta}(t)=n^{-1} \#\left\{i: d_{\theta}\left(X_{i}\right) \leq t\right\}$. 
There is a bijection between halfspaces and pairs $(\theta, t)$ for $\theta \in \mathbb{S}^{n-1}$ and $t \in \mathbb{R}$. In particular, for each $t$ and $\theta$, there is a halfspace $H_{\theta, t}$ such that $F_{\theta}(t)=\operatorname{Pr}\left(X \in H_{\theta, t}\right)$ and $F_{n, \theta}(t)=n^{-1} \#\left\{X^{(n)} \cap H_{\theta, t}\right\}$ (see Figure 1). In this case, $H_{\theta, t}$ is the halfspace whose bounding hyperplane passes through the point $t \cdot u_{\theta}$, whose bounding hyperplane has normal $u_{\theta}$, and the halfspace opens in the direction of $-u_{\theta}$. We can then rewrite the definition of halfspace depth as follows, cf [8]:

Proposition 3.3. For a point $q \in \mathbb{R}^{d}$, the halfspace depth of $q$ with respect to $X$ or $X^{(n)}$ is the following minimum over directions $\theta \in \mathbb{S}^{d-1}$ :

$$
\operatorname{HD}(q ; X)=\min _{\theta \in \mathbb{S}^{d-1}} F_{\theta}\left(d_{\theta}(q)\right) \quad \text { and } \quad \operatorname{HD}\left(q ; X^{(n)}\right)=\min _{\theta \in \mathbb{S}^{d-1}} F_{n, \theta}\left(d_{\theta}(q)\right) .
$$

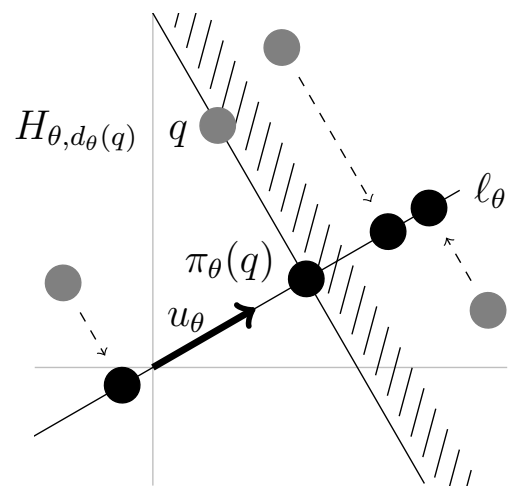

Figure 1: The number of points in the halfspace $H_{\theta, d_{\theta}(q)}$ (the unshaded halfspace) equals the number of points such that $d_{\theta}\left(X_{i}\right) \leq d_{\theta}(q)$. In the diagram, these are the points such that $\pi_{\theta}\left(X_{i}\right)$ is to the left of $\pi_{\theta}(q)$. A similar statement and diagram can be made for the random variable $X$.

\subsection{Spherical Covering}

As observed in Section 3.1, halfspace depth is related to the directions in a $(d-1)$ dimensional sphere. In our main result, we cover the $(d-1)$-dimensional sphere with small balls. The following result indicates how many of these neighborhoods are necessary.

Lemma 3.4 ([2, Corollary 1.2]). For any $0<\psi<\arccos \left(d^{-1 / 2}\right)$, there is an absolute constant $C_{1}$ such that the $(d-1)$-dimensional unit sphere can be covered by

$$
C_{1} \frac{\cos \psi}{\sin ^{d-1} \psi}(d-1)^{\frac{3}{2}} \ln \left(1+(d-1) \cos ^{2} \psi\right) \leq C_{1}\left(\frac{\sqrt{d}}{\psi}\right)^{d-1}(d-1)^{\frac{3}{2}} \ln (d)
$$

spherical balls of radius $\psi$ (i.e., the distance is measured by the central angle between the points).

The final inequality in this lemma follows from the facts that $\cos (\psi) \leq 1$ and for $0<$ $\psi<\arccos \left(d^{-1 / 2}\right), \sin (\psi) \geq \psi \cos (\psi)>d^{-1 / 2} \psi$. The $\sqrt{d}$ could be replaced by a constant by using a better bound on $\sin (x)$ such as $\sin (x) \geq x-\frac{x^{3}}{3 !}$. We leave the details to the interested reader. 


\subsection{The Dvoretzky-Kiefer-Wolfowitz Inequaulity}

In the one-dimensional case, the bounds in Proposition 2.1 can be improved with the Dvoretzky-Kiefer-Wolfowitz inequality:

Lemma 3.5 (See [6]). Let $Y$ be an $\mathbb{R}$-valued random variable, and let $\left(Y_{1}, Y_{2}, \cdots\right)$ be a sequence of iid random variables equal in distribution to $Y$. Let $F$ be the cdf of $Y$, and let $F_{n}$ be the empirical cdf, i.e., $F_{n}(t)=n^{-1} \#\left\{i \leq n: Y_{i} \leq t\right\}$. For each $\varepsilon>0$,

$$
\operatorname{Pr}\left(\sup _{t \in \mathbb{R}}\left|F(t)-F_{n}(t)\right| \geq \varepsilon\right) \leq 2 e^{-2 n \varepsilon^{2}} .
$$

In this paper, we use the Dvoretzky-Kiefer-Wolfowitz inequality on one-dimensional projections, as in Proposition 3.3, in order to improve the bound on the convergence rate of the sample halfspace depth.

\subsection{Lipschitz and Tail Behavior}

In this section, we define a few mild conditions which restrict the probability distributions that we consider.

Definition 3.6. The decay function of $X$ is the function $f(R)=\operatorname{Pr}(\|X\|>R)$. We say that $X$ decays exponentially if $f(R)=O\left(e^{-\lambda \frac{R^{2}}{2}}\right)$. In other words, there is a constant $C_{2}>0$ such that $\operatorname{Pr}(\|X\|>R) \leq C_{2} e^{-\lambda \frac{R^{2}}{2}}$ for $R>0$. We say that $\lambda$ is the (exponential) decay rate of $X$.

The standard multivariate normal with the origin the mean and covariance matrix $I$ decays exponentially for any $\lambda<1$.

Definition 3.7. We say that $X$ is Lipschitz continuous in projection if there is a constant $L_{\pi}>0$ such that for all $\theta \in \mathbb{S}^{d-1}, F_{\theta}$ is Lipschitz continuous with Lipschitz constant $L_{\pi}$. We say that $X$ is radially Lipschitz continuous if there is a constant $L_{\angle}$ such that for any fixed $t$, the function $F_{\theta}(t)$ is Lipschitz continuous as a function of $\theta$ on the $(d-1)$-dimensional sphere with Lipschitz constant $L_{L}$. We say that $L_{\pi}$ is the projection Lipschitz constant and $L_{L}$ is the radial Lipschitz constant for $X$.

Spherically symmetric distributions about the origin have $L_{L}=0$. These two Lipschitz constants indicate that as $\theta$ and $t$ change, $F_{\theta}(t)$ changes continuously ${ }^{2}$.

\footnotetext{
${ }^{2} L_{\pi}$ and $L_{\angle}$ can be bounded from above by the Lipschitz continuity constant of $\operatorname{Pr}(X \in H)$ in the space of all halfspaces $H$ in $\mathbb{R}^{d}$. We leave the details to the interested reader.

Note that these Lipschitz constants are based on the values corresponding to projections onto lines through the origin. We can, alternatively, consider projections onto the set of lines through any other point $P$. If both $L_{\angle}$ and $L_{\pi}$ are finite, then the corresponding Lipschitz constants for the point $P$ are also finite $\left(L_{\pi}\right.$ is unchanged and $L_{L}$ increases by at most $\|P\| L_{\pi}$ ). In the proof, $P$ is projected onto lines through the origin and Lemma 4.3 is applied. We, again, leave the details to the interested reader. Since the Lipschitz constants are finite at all points, we consider only projections onto lines through the origin for simplicity. Determining the point which attains the smallest value for $L_{L}$ remains an open question.
} 


\section{Main Inequality}

We begin this section by highlighting the difference between the previous approach and our technique. In Proposition 2.1, the convergence rates are computed over all halfspaces in $\mathbb{R}^{d}$, and the goal is to directly find an upper bound on

$$
\operatorname{Pr}\left(\sup _{H \in \mathcal{H}}\left|n^{-1} \#\left\{X^{(n)} \cap H\right\}-\operatorname{Pr}(X \in H)\right| \geq \varepsilon\right) .
$$

In our approach, however, we reorganize the halfspaces into one-dimensional families. In other words, we use the structure of the space of halfspaces in order to improve our bounds. In particular, our goal is to find bounds on the family of one-dimensional cdfs $F_{\theta}$ and $F_{n, \theta}$. More precisely, we find a uniform upper bound on the following probability:

$$
\operatorname{Pr}\left(\sup _{\substack{t \in \mathbb{R} \\ \theta \in \mathbb{S}^{d-1}}}\left|F_{\theta}(t)-F_{n, \theta}(t)\right| \geq \varepsilon\right) .
$$

This expression is the same as in Expression (1); we have rewritten it in this way to illustrate the idea behind the constructions of the one-dimensional families. By applying Proposition 3.3, when $\left|F_{\theta}(t)-F_{n, \theta}(t)\right| \leq \varepsilon$ for all $t$ and $\theta$, it follows that for all $q \in \mathbb{R}^{d}$, $\left|\operatorname{HD}(q ; X)-\operatorname{HD}\left(q ; X^{(n)}\right)\right| \leq \varepsilon$. The advantage of this approach is that since we have collected the halfspaces into one-dimensional families, we can apply the improved bounds of the Dvoretzky-Kiefer-Wolfowitz inequality.

Notation 4.1. Throughout the remainder of this section, we assume that $X$ has projection Lipschitz constant $L_{\pi}$ and radial Lipschitz constant $L_{L}$. In addition, we denote the decay function of $X$ by $f(R)$.

Our proof follows the following steps: (1) First, we quantify how small changes in $\theta$ affect the cdfs $F_{\theta}$ and $F_{n, \theta}$. (2) Second, we use this description on $F_{\theta}$ and $F_{n, \theta}$ to argue that it is sufficient to examine finitely many $\theta$ 's instead of the infinitely many possible $\theta$ 's in $\mathbb{S}^{d-1}$. (3) Finally, we apply the Dvoretzky-Kiefer-Wolfowitz inequality to each of these finitely many $\theta$ 's in order to uniformly bound the probability.

For our first step, we begin by collecting, for later convenience, a consequence of the Lipschitz condition and then provide a geometric description of the behavior of $d_{\theta}$ as $\theta$ changes. For notational convenience, for $\theta, \varphi \in \mathbb{S}^{d-1}$, we write $|\theta-\varphi|$ for the spherical distance between them, i.e., the central angle between the two points.

Observation 4.2. For all $\theta, \varphi \in \mathbb{S}^{d-1}$ and $t \in \mathbb{R},\left|F_{\theta}(t)-F_{\varphi}(t)\right| \leq L_{L}|\theta-\varphi|$.

Lemma 4.3. Let $\theta, \varphi \in \mathbb{S}^{d-1}$ and $x \in \mathbb{R}^{d}$. Then, $\left|d_{\theta}(x)-d_{\varphi}(x)\right| \leq\|x\| \cdot|\theta-\varphi|$.

Proof. Observe first that $\left|d_{\theta}(x)-d_{\varphi}(x)\right|=\left|\left\langle x, u_{\theta}-u_{\varphi}\right\rangle\right| \leq\|x\|\left\|u_{\theta}-u_{\varphi}\right\|$. Note that the vector $u_{\theta}-u_{\varphi}$ is the chord of an arc on the great circle between $\theta$ and $\varphi$; since chords are shorter than the arcs they cut off, and the length of the arc is $|\theta-\varphi|$, the result follows.

Lemma 4.3 allows us to bound $F_{n, \varphi}$ in terms of $F_{n, \theta}$. More precisely:

Corollary 4.4. Fix $R>0$ and $\theta, \varphi \in \mathbb{S}^{d-1}$. Suppose that for all $i \leq n,\left\|X_{i}\right\| \leq R$. Then,

$$
F_{n, \theta}(t-R|\theta-\varphi|) \leq F_{n, \varphi}(t) \leq F_{n, \theta}(t+R|\theta-\varphi|) .
$$


Proof. This result follows from Lemma 4.3, because we know that if $d_{\varphi}\left(X_{i}\right) \leq t$, then $d_{\theta}\left(X_{i}\right) \leq t+R|\theta-\varphi|$, and if $d_{\theta}\left(X_{i}\right) \leq t-R|\theta-\varphi|$, then $d_{\varphi}\left(X_{i}\right) \leq t$.

With Observation 4.2 and Corollary 4.4 in hand, we know how the distributions $F_{\theta}$ and $F_{n, \theta}$ vary as $\theta$ varies. This leads to the following proposition, which bounds the error between $F_{\theta}$ and $F_{n, \theta}$ as $\theta$ varies.

Proposition 4.5. Fix $R>0, \varepsilon>0, \delta>0$, and $\theta, \varphi \in \mathbb{S}^{d-1}$. Suppose that for all $i \leq n$, $\left\|X_{i}\right\| \leq R$. Suppose that $\sup _{t \in \mathbb{R}}\left|F_{\theta}(t)-F_{n, \theta}(t)\right|<\varepsilon(1+\delta)^{-1}$. Then,

$$
\sup _{t \in \mathbb{R}}\left|F_{\varphi}(t)-F_{n, \varphi}(t)\right|<\varepsilon(1+\delta)^{-1}+\left(L_{L}+L_{\pi} R\right)|\theta-\varphi| .
$$

Proof. By Corollary 4.4, we know that

$$
F_{n, \theta}(t-R|\theta-\varphi|)-F_{\varphi}(t) \leq F_{n, \varphi}(t)-F_{\varphi}(t) \leq F_{n, \theta}(t+R|\theta-\varphi|)-F_{\varphi}(t) .
$$

Then, it follows that

$$
\left|F_{n, \varphi}(t)-F_{\varphi}(t)\right| \leq \max \left\{\left|F_{n, \theta}(t-R|\theta-\varphi|)-F_{\varphi}(t)\right|,\left|F_{n, \theta}(t+R|\theta-\varphi|)-F_{\varphi}(t)\right|\right\} .
$$

We proceed to bound the first of the expression in the maximum (the other expression is similar). By the triangle inequality,

$$
\begin{aligned}
& \left|F_{n, \theta}(t-R|\theta-\varphi|)-F_{\varphi}(t)\right| \\
& \quad \leq\left|F_{n, \theta}(t-R|\theta-\varphi|)-F_{\theta}(t-R|\theta-\varphi|)\right|+\left|F_{\theta}(t-R|\theta-\varphi|)-F_{\theta}(t)\right|+\left|F_{\theta}(t)-F_{\varphi}(t)\right| .
\end{aligned}
$$

By assumption, the first expression is bounded from above by $\varepsilon(1+\delta)^{-1}$. By the projection Lipschitz constant, the second expression is bounded from above by $L_{\pi} R|\theta-\varphi|$. Finally, by Observation 4.2, the third expression is bounded from above by $L_{L}|\theta-\varphi|$. Combining these bounds, the result follows.

By choosing $|\theta-\varphi|$ to be sufficiently small, as in the following corollary, we can insist that the errors are all bounded from above by $\varepsilon$.

Corollary 4.6. Fix $R>0, \varepsilon>0, \delta>0$, and $\theta \in \mathbb{S}^{d-1}$. Suppose that for all $i \leq n$, $\left\|X_{i}\right\| \leq R$. Let $\varphi \in \mathbb{S}^{d-1}$ be such that $|\theta-\varphi|<\varepsilon \delta(1+\delta)^{-1}\left(L_{L}+L_{\pi} R\right)^{-1}$. Suppose that $\sup _{t \in \mathbb{R}}\left|F_{\theta}(t)-F_{n, \theta}(t)\right|<\varepsilon(1+\delta)^{-1}$. Then, $\sup _{t \in \mathbb{R}}\left|F_{\varphi}(t)-F_{n, \varphi}(t)\right|<\varepsilon$.

Corollary 4.6 completes the second main step of the proof and gives us a way to study neighborhoods on $\mathbb{S}^{d-1}$ instead of individual projections. In particular, if $\left\{\theta_{1}, \cdots, \theta_{k}\right\}$ satisfy the conditions of Corollary 4.6, then we have estimates on the error in the $k$ spherical balls of radius $\varepsilon \delta(1+\delta)^{-1}\left(L_{L}+L_{\pi} R\right)^{-1}$ centered at the $\theta_{i}$ 's. We now determine enough $\theta_{i}$ 's so that their neighborhoods cover the sphere and use the Dvoretzky-Kiefer-Wolfowitz bound in these directions to get our initial estimate on the error between the halfspace depth of a sample and the underlying distribution. In particular, 
Proposition 4.7. Fix $R>0, \varepsilon>0$, and $\delta>0$. Suppose that $\varepsilon \delta(1+\delta)^{-1}\left(L_{\angle}+L_{\pi} R\right)^{-1}<$ $\arccos \left(d^{-1 / 2}\right)$. Then,

$$
\begin{gathered}
\operatorname{Pr}\left(\sup _{q \in \mathbb{R}^{d}}\left|\operatorname{HD}(q ; X)-\operatorname{HD}\left(q ; X^{(n)}\right)\right| \geq \varepsilon\right) \leq \\
2 C_{1}\left(\frac{(1+\delta)\left(L_{\angle}+L_{\pi} R\right) \sqrt{d}}{\varepsilon \delta}\right)^{d-1}(d-1)^{\frac{3}{2}} \ln (d) e^{-2 n \varepsilon^{2}(1+\delta)^{-2}}+n f(R) .
\end{gathered}
$$

Proof. Observe first, that, in each of the results above, we require that for all $i \leq n,\left\|X_{i}\right\| \leq$ $R$. Since there are $n$ sample points, the probability that any point fails this inequality is bounded from above by $n f(R)$.

Next, observe that if $\theta \in \mathbb{S}^{d-1}$ is such that $\sup _{t \in \mathbb{R}}\left|F_{\theta}(t)-F_{n, \theta}(t)\right|<\varepsilon(1+\delta)^{-1}$, then all $\varphi \in \mathbb{S}^{d-1}$ within a spherical ball of radius $\varepsilon \delta(1+\delta)^{-1}\left(L_{L}+L_{\pi} R\right)^{-1}$ satisfy the conditions of Corollary 4.6. Therefore, we cover the $(d-1)$-dimensional sphere with balls of radius $\varepsilon \delta(1+\delta)^{-1}\left(L_{\angle}+L_{\pi} R\right)^{-1}$. By Lemma 3.4, $C_{1}\left((1+\delta)\left(L_{\angle}+L_{\pi} R\right) \sqrt{d}\right)^{d-1}(\varepsilon \delta)^{1-d}(d-1)^{\frac{3}{2}} \ln (d)$ such spherical balls are required. Fix $\theta$ to be the center of one of these spherical balls. Applying the Dvoretzky-Kiefer-Wolfowitz inequality, Lemma 3.5, it follows that

$$
\operatorname{Pr}\left(\sup _{t \in \mathbb{R}}\left|F_{\theta}(t)-F_{n, \theta}(t)\right| \geq \varepsilon(1+\delta)^{-1}\right) \leq 2 e^{-2 n \varepsilon^{2}(1+\delta)^{-2}} .
$$

Since the spherical balls cover $\mathbb{S}^{d-1}$, for any $\varphi \in \mathbb{S}^{d-1}$, there is some spherical ball with center $\theta$ such that $|\theta-\varphi|<\varepsilon \delta(1+\delta)^{-1}\left(L_{\angle}+L_{\pi} R\right)^{-1}$. Hence, Inequality (5) indicates when Corollary 4.6 can be applied to conclude that $\sup _{t \in \mathbb{R}}\left|F_{\varphi}(t)-F_{n, \varphi}(t)\right|<\varepsilon$. By applying Inequality (5) to each ball, the result follows.

\section{Examples}

In this section, we apply Proposition 4.7 to several examples. We take special care to develop explicit bounds on the Lipschitz constants whenever possible. In the case where $X$ decays exponentially, Proposition 4.7 results in a bound which improves upon the best known convergence rates in Proposition 2.1.

Theorem 5.1. Fix $\varepsilon>0$, and suppose that $X$ decays exponentially with decay rate $\lambda$. Then, for $n$ sufficiently large,

$$
\begin{aligned}
\operatorname{Pr}\left(\sup _{q \in \mathbb{R}^{d}} \mid\right. & \left.\operatorname{HD}(q ; X)-\operatorname{HD}\left(q ; X^{(n)}\right) \mid \geq \varepsilon\right) \leq \\
& \left(2 C_{1}\left(\frac{\left(L_{\angle} \sqrt{\lambda}(n+1)+2 L_{\pi} n^{\frac{3}{2}} \varepsilon\right) \sqrt{d}}{\varepsilon \sqrt{\lambda}}\right)^{d-1}(d-1)^{\frac{3}{2}} \ln (d)+C_{2} n\right) e^{4} e^{-2 n \varepsilon^{2}} .
\end{aligned}
$$

Proof. Since $X$ decays exponentially with decay rate $\lambda$, let $C_{2}>0$ be the constant such that for $R>0, \operatorname{Pr}(\|X\|>R) \leq C_{2} e^{-\lambda \frac{R^{2}}{2}}$. Additionally, throughout the remainder of this proof, 
we assume that $n$ is large enough so that the two inequalities $\varepsilon(n+1)^{-1}\left(L_{\angle}+L_{\pi} \varepsilon \frac{2 n^{3 / 2}}{\sqrt{\lambda}(n+1)}\right)^{-1}<$ $\arccos \left(d^{-1 / 2}\right)$ and $2 \varepsilon n^{\frac{3}{2}}>\sqrt{\lambda}(n+1)$ hold.

We consider the following two substitutions: (1) $R=\varepsilon \frac{2 \sqrt{n}}{\sqrt{\lambda}(1+\delta)}$ and (2) $\delta=\frac{1}{n}$. With these substitutions, and the first inequality on $n$ above, it follows that the assumptions of Proposition 4.7 apply. The choice of $R$ makes the two exponential portions of the bound identical, i.e., $e^{-2 n \varepsilon^{2}(1+\delta)^{-2}}$. We now show that with the choice of $\delta$ above, $e^{-2 n \varepsilon^{2}(1+\delta)^{-2}}$ is bounded from above by a constant times $e^{-2 n \varepsilon^{2}}$, matching the exponent of Inequality (2) of Proposition 2.1. In particular, $n(1+\delta)^{-2}=n-\frac{2 n^{2}+n}{n^{2}+2 n+1}$. The quotient is bounded from below by -2 for $n$ positive. Therefore, $n(1+\delta)^{-2} \geq n-2$ and $e^{-2 n \varepsilon^{2}(1+\delta)^{-2}}<e^{-2 n \varepsilon^{2}} e^{4 \varepsilon}$. Since $\varepsilon$ is at most $1, e^{4 \varepsilon} \leq e^{4}$ and the result follows.

Theorem 1.3 then follows from Theorem 5.1 by observing that the highest power of $n$ which appears is $n^{\frac{3}{2}(d-1)}$. We can compare this result with the known convergence rates in Proposition 2.1. The exponential in Inequality (1) is much larger (the exponent is a negative number with smaller magnitude) than what appears in Theorem 5.1 and Theorem 1.3, so our bound is tighter. On the other hand, the coefficient of $e^{-2 n \varepsilon^{2}}$ in Inequality (2) is polynomial in $n$ of degree $2 d+2$, which is a factor of $n^{\frac{1}{2} d+\frac{7}{2}}$ times larger than our bound.

In addition, this bound is reasonably tight, as seen in the following example:

Example 5.2. Fix $0<\varepsilon<\frac{1}{2}$ and assume that $X$ is distributed according to a standard normal distribution in $\mathbb{R}^{d}$ with covariance matrix $I$. Then the halfspace depth of the origin is $\frac{1}{2}$, with respect to the distribution. Therefore, every halfspace $H$ which passes through the origin has $\operatorname{Pr}(X \in H)=\frac{1}{2}$.

Turning to a sample $X^{(n)}$, the error between $\frac{1}{2}$ and halfspace depth of the origin with respect to the sample is at least $\varepsilon$ if there exists a halfspace containing the origin and at most $n / 2-n \varepsilon$ sample points. Therefore, whether a sample is in $H$ is governed by a binomial distribution with $p=\frac{1}{2}$.

We bound the probability that a halfspace $H$ contains exactly $n / 2-n \varepsilon$ sample points (assuming $n / 2-n \varepsilon$ is an integer). The probability that $H$ contains exactly $n / 2-n \varepsilon$ sample points is $2^{-n}\left(\begin{array}{c}n \\ n / 2-n \varepsilon\end{array}\right)$. Using the bounds on Stirling's formula in [19] and observing that the exponential error terms approach 1 as $n$ increases, we have that, up to a constant factor,

$$
\frac{1}{2^{n}}\left(\begin{array}{c}
n \\
n / 2-n \varepsilon
\end{array}\right) \gtrsim \frac{1}{2^{n}} \frac{1}{\sqrt{2 \pi n}} \frac{1}{\left(\frac{1}{4}-\varepsilon^{2}\right)^{n / 2+1 / 2}}\left(\frac{1-2 \varepsilon}{1+2 \varepsilon}\right)^{n \varepsilon}=\sqrt{\frac{2}{\pi n}} e^{-\ln \left(1-4 \varepsilon^{2}\right)\left(\frac{n}{2}+\frac{1}{2}\right)+\ln \left(\frac{1-2 \varepsilon}{1+2 \varepsilon}\right) n \varepsilon}
$$

Taking the first order expansions of the power series (assuming $\varepsilon$ is small enough so that the higher order terms are insignificant), the exponent of the exponential is $4 \varepsilon^{2}\left(\frac{n}{2}+\frac{1}{2}\right)-4 n \varepsilon^{2}$. Since $\varepsilon$ is treated as a constant here, this probability is, up to a constant factor, $\frac{1}{\sqrt{n}} e^{-2 n \varepsilon^{2}}$ whose exponential matches the one in our bound.

For elliptically symmetric distributions, the bound in Theorem 5.1 can be made even simpler.

Example 5.3. Consider the case where $X$ is distributed according to an elliptically symmetric distribution with pdf $f(x)=\operatorname{det}(\Sigma)^{-\frac{1}{2}} \psi\left((x-\mu)^{T} \Sigma^{-1}(x-\mu)\right)$ where $x$ and $\mu$ are 
in $\mathbb{R}^{d}, \Sigma$ is a positive definite symmetric $d \times d$ matrix, and $\psi:[0, \infty) \rightarrow[0, \infty)$ (see [4]). Since $\Sigma$ is positive definite and halfspace depth is affine invariant (see [24]), we can use the eigendecomposition of $\Sigma$ to construct an affine transformation which transforms $X$ into a spherically symmetric distribution about the origin. Therefore, we assume that $X$ is a spherical distribution whose pdf is $\psi\left(\sum y_{i}^{2}\right)$. Like all distributions which are spherically symmetric about the origin, $L_{L}=0$. Additionally, since $F_{\theta}$ is independent of $\theta$, the projection Lipschitz constant can be found as the maximum of the density of the derivative of $F_{\theta}(t)$ with respect to $t$, i.e., $L_{\pi}=\sup _{t \in \mathbb{R}}\left|\int_{\mathbb{R}^{d-1}} \psi\left(t^{2}+x_{1}^{2}+\cdots+x_{d-1}^{2}\right) d x_{1} \cdots d x_{d-1}\right|$. Finally, the decay function of $X$ is $\int_{R}^{\infty} r^{d-1} \psi\left(r^{2}\right) d r$.

In particular cases, we can derive more precise bounds and constants.

Example 5.4. Consider the case where $X$ is distributed according to a standard normal distribution in $\mathbb{R}^{d}$ centered at the origin with covariance matrix $I$. In this case, the pdf of $X$ is $(2 \pi)^{-d / 2} e^{-\frac{1}{2} \sum x_{i}^{2}}$. Via spherical integration, it follows that $\operatorname{Pr}(\|X\|>R)=O\left(R^{d-2} e^{-\frac{R^{2}}{2}}\right)$, so the decay rate of $X$ can be any positive constant less than 1 . Additionally, for any $\theta, F_{\theta}$ is the cdf of a standard normal distribution in one variable, hence the Lipschitz constant for $F_{\theta}$ is the maximum of the pdf of a standard normal distribution in one variable, $L_{\pi}=\frac{1}{\sqrt{2 \pi}}$.

Example 5.5. For the two-dimensional normal, we can say even more. More precisely, assume that $X$ is distributed according to a bivariate normal centered at the origin with covariance matrix $I$. The bounds above can be made even sharper: We can cover a circle using at most $\frac{\pi}{w}+1$ intervals of width $2 w$, and $\operatorname{Pr}(\|X\|>R)=e^{-\frac{1}{2} R^{2}}$. Therefore, in this case, the bound in Theorem 5.1 has a smaller constant, and, explicitly, the bound becomes

$$
\operatorname{Pr}\left(\sup _{q \in \mathbb{R}^{2}}\left|\operatorname{HD}(q ; X)-\operatorname{HD}\left(q ; X^{(n)}\right)\right| \geq \varepsilon\right) \leq\left(2 \sqrt{2 \pi} n^{\frac{3}{2}}+n+2\right) e^{4} e^{-2 n \varepsilon^{2}}
$$

On the other hand, in the two-dimensional case, the function $m$ for Proposition 2.1 can also be determined explicitly. The largest number of subsets of a set of size $n$ occurs when the points of $n$ are in convex position, i.e., form the vertices of a convex polygon. In this case, there are $n^{2}-n+2$ subsets formed from intersections with halfspaces, so $m(r)=r^{2}-r+2$. Even with this smaller degree polynomial for $m$, our bounds are still an improvement by a factor of $\sqrt{n}$.

\section{Conclusion}

The results in this paper illustrate how, using the geometry and structure of halfspace depth and $\mathbb{R}^{d}$, one can achieve better convergence bounds for the sample version of halfspace depth, as compared to usual Glivenko-Cantelli bounds. With these improved bounds, one can improve estimates on the quality of the halfspace median statistic or halfspace depth contours (see [16] and [1]). The approach in this paper is to reduce a high-dimensional problem into a collection of one-dimensional problems, and use the solutions of these one-dimensional problems to improve the convergence rates or the original problem. This approach is related to the ideas of the projection pursuit in [7] and [18]; it is possible that incorporating such 
techniques may further improve the convergence rates of halfspace depth; we leave such improvements as future work.

The authors would like to thank the anonymous referees and the authors' colleagues at Clemson University, in particular, Billy Bridges, Brian Fralix, Peter Kiessler, and June Luo, for their constructive feedback on earlier versions of this work.

[1] Greg Aloupis. Geometric measures of data depth. In Data depth: robust multivariate analysis, computational geometry and applications, volume 72 of DIMACS Series on Discrete Mathematics and Theoretical Computer Science, pages 147-158. American Mathematical Society, Providence, RI, 2006.

[2] Károly Böröczky, Jr. and Gergely Wintsche. Covering the sphere by equal spherical balls. In Discrete and computational geometry, volume 25 of Algorithms Combinatorics, pages 235-251. Springer, Berlin, 2003.

[3] Michael A. Burr, Eynat Rafalin, and Diane L. Souvaine. Dynamic maintenance of halfspace depth for points and contours. Technical Report arXiv:1109.1517 [cs.CG], arXiv, 2011.

[4] M. A. Chmielewski. Elliptically symmetric distributions: a review and bibliography. International Statistical Review. Revue International de Statistique, 49(1):67-74, 1981.

[5] Luc Devroye. Upper and lower class sequences for minimal uniform spacings. Zeitschrift für Wahrscheinlichkeitstheorie und Verwandte Gebiete, 61(2):237-254, 1982.

[6] Luc Devroye and Gábor Lugosi. Combinatorial methods in density estimation. Springer Series in Statistics. Springer-Verlag, New York, 2001.

[7] Persi Diaconis and David Freedman. Asymptotics of graphical projection pursuit. The Annals of Statistics, 12(3):793-815, 1984.

[8] David L. Donoho and Miriam Gasko. Breakdown properties of location estimates based on halfspace depth and projected outlyingness. The Annals of Statistics, 20(4):18031827, 1992.

[9] Richard M. Dudley. Balls in $\mathbb{R}^{k}$ do not cut all subsets of $k+2$ points. Advances in Mathematics, 31(3):306-308, 1979.

[10] Lutz Dümbgen. Limit theorems for the simplicial depth. Statistics \& Probability Letters, 14(2):119-128, 1992.

[11] Rainer Dyckerhoff and Pavlo Mozharovskyi. Exact computation of the halfspace depth. Computational Statistics \& Data Analysis, 98:19 - 30, 2016.

[12] Xuming He and Gang Wang. Convergence of depth contours for multivariate datasets. The Annals of Statistics, 25(2):495-504, 1997.

[13] Joseph L. Hodges, Jr. A bivariate sign test. Annals of Mathematical Statistics, 26:523$527,1955$. 
[14] Jeankyung Kim. Rate of convergence of depth contours: with application to a multivariate metrically trimmed mean. Statistics $\&$ Probability Letters, 49(4):393-400, 2000.

[15] Regina Y. Liu. Data depth: center-outward ordering of multivariate data and nonparametric multivariate statistics. In Michael G. Akritas and Dimitris N. Politis, editors, Recent advances and trends in nonparametric statistics, pages 155-167. Elsevier B. V., Amsterdam, 2003.

[16] Regina Y. Liu, Jesse M. Parelius, and Kesar Singh. Multivariate analysis by data depth: descriptive statistics, graphics and inference. The Annals of Statistics, 27(3):783-858, 1999.

[17] Jean-Claude Massé. Asymptotics for the Tukey depth process, with an application to a multivariate trimmed mean. Bernoulli, 10(3):397-419, 62004.

[18] Elizabeth S. Meckes. Quantitative asymptotics of graphical projection pursuit. Electronic Communications in Probability, 14:176-185, 2009.

[19] Herbert Robbins. A remark on Stirling's formula. The American Mathematical Monthly, 62:26-29, 1955.

[20] Peter J. Rousseeuw and Mia Hubert. Statistical depth meets computational geometry: a short survey. Technical Report arXiv:1508.03828 [stat.ME], arXiv, 2015.

[21] Galen R. Shorack and Jon A. Wellner. Empirical processes with applications to statistics. Wiley Series in Probability and Mathematical Statistics: Probability and Mathematical Statistics. John Wiley \& Sons, Inc., New York, 1986.

[22] John W. Tukey. Mathematics and the picturing of data. In Proceedings of the International Congress of Mathematicians (Vancouver, B. C., 1974), Vol. 2, pages 523-531. Canadian Mathematical Congress, Montreal, Que., 1975.

[23] V. N. Vapnik and A. Ja. Cervonenkis. The uniform convergence of frequencies of the appearance of events to their probabilities. Akademija Nauk SSSR. Teorija Verojatnosteu i ee Primenenija, 16:264-279, 1971.

[24] Yijun Zuo and Robert Serfling. General notions of statistical depth function. The Annals of Statistics, 28(2):461-482, 2000.

[25] Yijun Zuo and Robert Serfling. Structural properties and convergence results for contours of sample statistical depth functions. The Annals of Statistics, 28(2):483-499, 2000 . 